https://doi.org/10.15407/ujpe64.10.933

C.E. ZASPEL, ${ }^{1}$ G.M. WYSIN, ${ }^{2}$ B.A. IVANOV $^{3,4}$

${ }^{1}$ Department of Environmental Science, University of Montana-Western (Dillon, MT 59725 USA)

2 Department of Physics, Kansas State University (Manhattan, KS 66506 USA)

${ }^{3}$ Institute of Magnetism, Nat. Acad. of Sci. of Ukraine

(36 b, Vernadsky St., 03142 Kyiv, Ukraine)

${ }^{4}$ Faculty of Radio Physics, Electronics and Computer Systems, Taras Shevchenko National University of Kyiv (4G, Academician Glushkov Prosp., Kyiv 03187, Ukraine)

\title{
MAGNETIC CHIRAL SOLITONS STABILIZED BY OERSTED FIELD AT A THIN-FILM NANOCONTACT WITH ELECTRIC CURRENT
}

\begin{abstract}
Static magnetic solitons in a thin film such as skyrmions are metastable states that can be stabilized through a balance of the exchange interaction and various relativistic interactions. One of the most effective stabilizing terms is the antisymmetric exchange along with others such as magnetostatic interactions in confined structures, as well as a current-carrying nanocontact on a thin ferromagnetic film. In this article, the effect of a nanocontact current on the energies of both topological (T-type) and nontopological (NT-type) solitons has been investigated. Without an antisymmetric exchange interaction, the Oersted field from a nanocontact can stabilize both soliton types with the NT soliton as the ground state. With the antisymmetric exchange, there is a critical nanocontact current, where the T soliton becomes the ground state.

Ke ywords: skyrmion, Oersted field, nanocontact.
\end{abstract}

\section{Introduction}

Chiral magnetic skyrmions are localized soliton-like structures having topological charge $(Q= \pm 1)$ in quasi-two-dimensional (2D) magnetic systems. Their topological properties and small size make skyrmions useful for nanoscale applications such as magnetic memory, logic devices [1], and high frequency spintorque oscillators [2-6]. One of the first exact magnetic skyrmion solutions was obtained by Belavin and Polyakov [7] for the 2D isotropic Heisenberg model in the continuum approximation. A special case of this solution is simply the mapping of magnet's plane onto the magnetization sphere having energy independent of the soliton size. This soliton is topologically stable, but it becomes unstable, if a small easyaxis anisotropy is accounting for, which results in a collapse of the structure. Indeed, according to the Hobart-Derrik theorem, any 2D energy density with only second-order magnetic gradients cannot support static solitons. However, it has been shown that the

(C) C.E. ZASPEL, G.M. WYSIN, B.A. IVANOV, 2019

ISSN 2071-0194. Ukr. J. Phys. 2019. Vol. 64, No. 10 dynamic precession of the magnetization about an easy axis will support stable dynamic solitons, see [8-11] and [12] for the review. Recently, it has been shown that such precessional skyrmions can be stable in a nanocontact carrying a spin polarized current [13].

In general, the stabilization can be achieved through a delicate balance of the exchange interaction and various relativistic or exchange-relativistic interactions. It was initially shown $[14,15]$ that the stabilization is possible with the Dzialoshinsky-Moria (DM) interaction in non-centrosymmetric magnets and at interfaces [16]. This interaction can favor the formation of skyrmion ground and metastable states, as well as skyrmion lattices [16-18]. In addition to the DM interaction, other stabilization mechanisms have been investigated. First, the competing interactions from the frustrated exchange $[15,19$, 20 ], which can support large-radius solitons in twodimensional systems, can be mentioned. Competing interactions from magnetic dipolar interactions [2124] have also been shown to support skyrmion struc- 


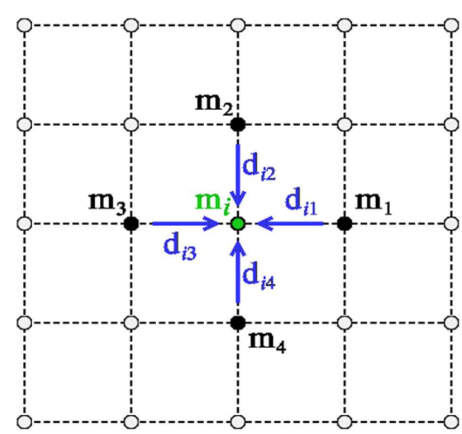

Fig. 1. Square lattice structure indicating the DM vector orientation

tures in confined nanostructures. More recently, it has been demonstrated that the curvature on a film surface [25] can provide the chirality to stabilize skyrmions, where the soliton size is related to the surface curvature. Finally, the specially designed patterned thin ferromagnetic films can provide the chirality necessary for the formation of magnetic vortices [26] and skyrmions [27].

In this article, the single soliton formation at a nanocontact is investigated, and it is remarked that both topological ( $\mathrm{T}$ ) with $Q= \pm 1$ and nontopological (NT) solitons with $Q=0$ are stable even in the absence of a DM interaction. The T soliton is defined as a complete mapping of the plane of a $2 \mathrm{D}$ magnet onto the magnetization sphere $\mathbf{M}^{2}=M_{S}^{2}$, where $\mathbf{M}$ is the magnetization and $M_{S}$ is the saturation magnetization. The NT soliton is characterized by the incomplete mapping leaving a hole in the magnetization sphere. If the DM interaction is absent, it is shown that the NT soliton is always the lower energy state, than the T soliton, but they both are structures stable against small perturbations. Including the DM interaction in the energy density, it is found that there is a critical nanocontact current, above which the $\mathrm{T}$ soliton will have the lower energy. This effect has potential device applications, since the energies of $\mathrm{T}$ and NT solitons can be manipulated through an external current. First, the magnetization distribution in the solitons is obtained by the minimization of the continuum approximate energy and the numerical solution of the corresponding Euler-Lagrange equations. These solutions are then used to calculate the energy by a numerical integration of the energy density in the continuum approximation.

We begin with the energy in the continuous approximation for a magnetic thin film with a nanocontact of radius $r_{0}$ carrying a current $I$. It is also assumed that the film is thin so that the magnetization is independent of the coordinate in the direction perpendicular to the plane, i.e., the distribution of the magnetization is two-dimensional. First, the exchange energy is

$W_{\mathrm{Ex}}=A L \int(\nabla \mathbf{m})^{2} d^{2} r$

where $A$ is the inhomogeneous exchange constant, $L$ is the film thickness, and $\mathbf{m}=\mathbf{M} / M_{S}=$ $=(\sin (\theta) \cos (\varphi), \sin (\theta) \sin (\varphi), \cos (\theta))$ is the normalized magnetization, where $\theta$ and $\varphi$ are the polar and azimuthal angles of the magnetization in the film. The easy-axis anisotropy energy is,

$W_{\mathrm{A}}=K L \int \sin ^{2} \theta d^{2} r$

where $K>0$ is the anisotropy constant. In the expression for the Oersted and DM energies, it is assumed that a Bloch skyrmion is centered at the nanocontact with $\varphi=\chi+\pi / 2$, and $\theta(r)$ is a function of $r$ only in polar $(r, \chi)$ coordinates. Then the Oersted energy for a nanocontact of radius $r_{0}$ carrying a current $I$ is

$W_{\text {Oe }}=-\frac{\mu_{0} M_{S} I}{2 \pi} L \int f(r) \sin \theta d^{2} r$,

where $f(r)=r /\left(r_{0}^{2}\right)$ for $r<r_{0}$, and $f(r)=1 / r$ for $r \geq r_{0}$. The DM energy supported Bloch skyrmions is $[14,15]$

$W_{D M}=D M_{S}^{2} L \int\left(\theta_{r}+\frac{1}{r} \sin \theta \cos \theta\right) d^{2} r$,

corresponding to the DM vector illustrated on a discrete lattice in Fig. 1. Here, the $r$ subscript indicates a radial derivative.

Next, the minimization of the energy density with respect to $\theta(r)$ gives the following differential equation:

$\theta_{r r}+\frac{1}{r} \theta_{r}-\left(\frac{1}{r^{2}}+\frac{1}{l_{K}^{2}}\right) \sin \theta \cos \theta+$

$+\frac{f(r)}{l_{\mathrm{Oe}}} \cos \theta+\frac{2}{r l_{\mathrm{DM}}} \sin ^{2} \theta=0$.

Here, we have introduced the characteristic lengths corresponding to the various contributions to the energy, $l_{K}^{2}=A / K, l_{\mathrm{DM}}=2 A /\left(\mathrm{DM}_{S}^{2}\right)$, and $l_{\mathrm{Oe}}=$ 
$=4 \pi A /\left(\mu_{0} M_{S} I\right)$. The function $\theta(r)$ of a soliton is obtained through the numerical solution of Eq. 5 using the shooting method for the boundary condition, $\theta(0)=\pi$ for the $\mathrm{T}$ soliton, and $\theta(0)=0$ for the $\mathrm{NT}$ soliton. It is remarked also that $\theta \rightarrow 0$ as $r \rightarrow \infty$ on the thin film. For large values of $r$, Eq. 5 is linearized, and the large- $r$ solution is obtained:

$\theta=\frac{l_{K}^{2}}{l_{\mathrm{Oe}} r}$.

This large- $r$ form for the soliton structure has a significant contribution to the soliton energy. A magnetic soliton typically has a finite energy independent of the system size, which is indeed the case for all of the above terms in the total energy, except for the Oersted term, where the energy density has a $1 / r^{2}$ dependence. Therefore, in the following, the soliton energy is expressed as $W\left(r_{\mathrm{c}}\right)=W_{1}\left(r_{\mathrm{c}}\right)+W_{2}\left(r_{\mathrm{c}}\right)$, where $W_{1}$ is the energy obtained by the numerical solution of Eq. 5 in all contributions to the energy and by the integration of this solution to a cut-off upper limit, $r_{\mathrm{c}}$. The second contribution to the energy, $W_{2}$, is obtained by the analytic calculation of the energy integrals using Eq. 6 from $r_{\mathrm{c}}$ as the lower limit to an upper limit, $R$. Then the cut-off is chosen so that the total energy, $W\left(r_{\mathrm{c}}\right)$, has no significant dependence on $r_{\mathrm{c}}$.

There are two types of solitons investigated by the solution of Eq. 5: the first is a skyrmion or topological soliton, where $\theta=\pi$ at $r=0$, and the second is a nontopological soliton, where $\theta=0$ at $r=0$. It is also remarked that the $W_{2}$ contribution to the energy is independent of the soliton type, so only the $W_{1}$ contribution will be presented for each type. Let us first solve Eq. 5 in the case where the DM interaction is absent, and the chirality is provided by the Oersted field. The solutions are obtained using typical parameters of skyrmion-bearing thin films, $A=16$ $\mathrm{pJ} / \mathrm{m}$ and $M_{S}=1.1 \mathrm{MA} / \mathrm{m}$.

First, we consider these two types of soliton structures, a topological $(\mathrm{T})$ soliton and a nontopological (NT) soliton without the DM interaction. For currents of $20 \mathrm{~mA}$ and $100 \mathrm{~mA}$ with $l_{K}=20 \mathrm{~nm}$, these two soliton structures are illustrated in Fig. 2. At the high current, the ring-like region, where the soliton has an approximately planar $(\theta=\pi / 2)$ vortex structure, is present. Moreover, it is also seen that significant structural differences between the $\mathrm{T}$ and $\mathrm{NT}$ solitons are only observed for $r<100 \mathrm{~nm}$. The $W_{1}$

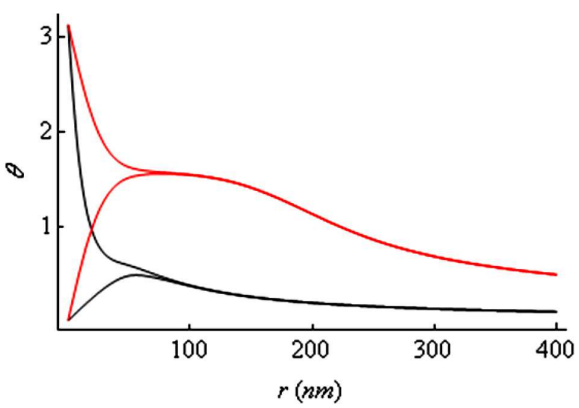

Fig. 2. T and NT soliton structures for a nanocontact of the radius $r_{0}=50 \mathrm{~nm}$ with currents of $100 \mathrm{~mA}$ (gray) and $20 \mathrm{~mA}$ (black)

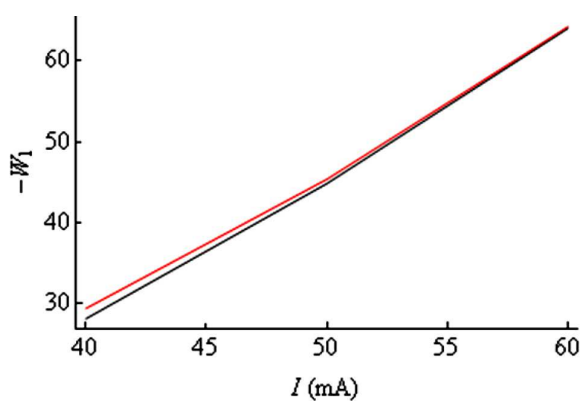

Fig. 3. Negative energy of T solitons (black) and NT solitons (gray) versus the current without the DM interaction. Here and below in Fig. 4, the energy is measured in units of $A L$, where $A$ is the exchange constant, and $L$ is the film thickness

values of both soliton structures with $r_{\mathrm{c}}=400 \mathrm{~nm}$ are calculated by the numerical integration of all nonzero contributions to the energy with the results shown in Fig. 3. Here, it is noticed that the NT soliton energy is always lower than the T soliton energy, although these energies become very close at high current values.

Finally, the combined effect of the DM and Oersted interactions is considered. Here, it is necessary to begin with a small enough DM interaction and nanocontact current combination so that the cycloid states will not be present. For the parameters considered here, this implies that $l_{\mathrm{DM}} \geq 40 \mathrm{~nm}$. The current-dependent soliton energies for the parameters, $l_{\mathrm{DM}}=40 \mathrm{~nm}$ and $l_{K}=20 \mathrm{~nm}$ are seen in Fig. 4. Here, we note that there is a critical current, $I_{\mathrm{c}}=28.9 \mathrm{~mA}$, where the $\mathrm{T}$ soliton energy becomes lower than the NT soliton energy. For smaller values of the DM interaction or larger values of $l_{\mathrm{DM}}$, a larger critical current is required. The DM-dependence of the critical current is seen in Fig. 5. Regarding the question of stability of both soliton types, prelimi- 


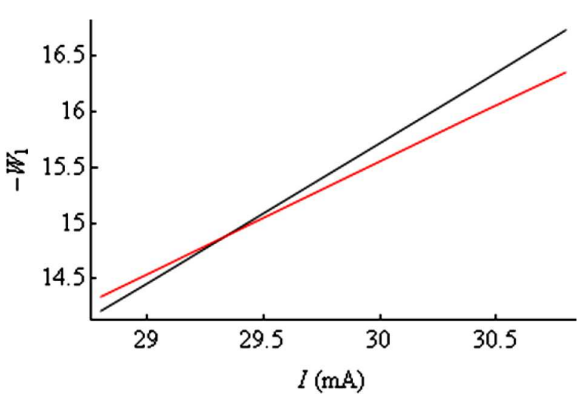

Fig. 4. Soliton energy versus the current for $\mathrm{T}$ solitons (black) and NT solitons (gray) in the presence of the DM interaction

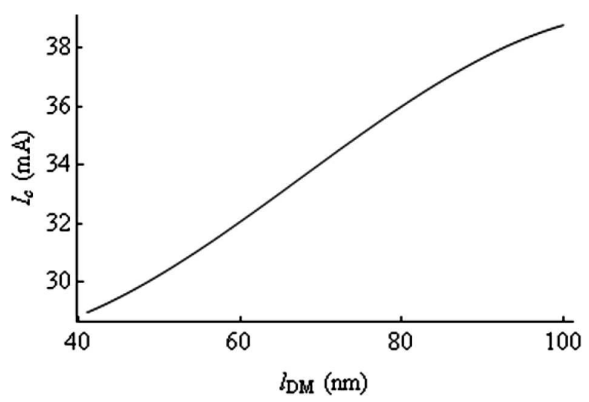

Fig. 5. Critical current versus $l_{\mathrm{DM}}$

nary numerical simulations indicate that both $\mathrm{T}$ and NT solitons are stable against small perturbations for the parameter ranges considered here.

In conclusion, as expected, a skyrmion can be formed in an Oersted field without the DM interaction. However, the skyrmion is not necessarily the lowest-energy soliton. A combination of the DM interaction and an Oersted field will result in the skyrmion having the lowest energy providing an outside method to manipulate soliton energies through a nanocontact. In addition to the skyrmion (T-soliton) and the NT soliton, it is also possible to find skyrmionium structures having the zero topological charge, as well as cycloid states above critical DM and nanocontact current values, but these will be the subject of a further research.

The work was sponsored by the National Academy of Sciences of Ukraine (grant No.0106U000594) and the Ministry of Education and Science of Ukraine (grants Nos. 0109U002069 and 0109U001151).

1. A. Fert, N. Reyren, V. Cros. Magnetic skyrmions: advances in physics and potential applications. Nat. Rev. Mater. 2, 17031 (2017).

2. L. Liu, C.-T. Chen, J.Z. Sun. Spin Hall effect tunnelling spectroscopy. Nat. Phys. 10, 561 (2014).

\section{6}

3. X.Z. Yu, N. Kanazawa, W.Z. Zhang, T. Nagai, T. Hara, K. Kimoto, Y. Matsui, Y. Onose, Y. Tokura. Skyrmion flow near room temperature in an ultralow current density. Nat. Comm. 3, 988 (2012).

4. F. Jonietz, S. Muhlbauer, C. Pfleiderer, A. Neubauer, W. Munzer, A. Bauer, T. Adams, R. Georgii, P. Boni, R.A. Duine, K. Everschor, M. Garst, A. Rosch. Spin Transfer Torques in MnSi at Ultralow Current Densities. Science 330, 1648 (2010).

5. J. Iwasaki, M. Mochizuki, N. Nagaosa. Current-induced skyrmion dynamics in constricted geometries. Nat. Nanotechnol. 8, 742 (2013).

6. K. Litzius, I. Lemesh, B. Kruger, P. Bassirian, L. Caretta, K. Richter, F. Buttner, K. Sato, O.A. Tretiakov, J. Förster, R.M. Reeve, M. Weigand, I. Bykova, H. Stoll, G. Schulz, G.S.D. Beach, M. Klaui. Skyrmion Hall effect revealed by direct time-resolved X-ray microscopy. Nat. Phys. 13, 170 (2017).

7. A.A. Belavin, A.M. Polyakov. Metastable states of twodimensional isotropic ferromagnets. JETP Lett. 22, 245 (1975).

8. A.S. Kovalev, A.M. Kosevich, K.V. Maslov. Magnetic vortex - topological soliton in a ferromagnet with an easy-axis anisotropy. JETP Lett. 30, 296 (1979).

9. V.P. Voronov, B.A. Ivanov, A.M. Kosevich. Two-dimensional dynamic (topological) solitons in ferromagnets $Z h$. Eksp. Teor. Fiz. 84, 2235 (1983).

10. B.A. Ivanov, V.A. Stefanovich. Two-dimensional small-radius solitons in magnets. Zh. Eksp. Teor. Fiz. 91, 638 (1986).

11. D.D. Sheka, B.A. Ivanov, F.G. Mertens. Internal modes and magnon scattering on topological solitons in twodimensional easy-axis ferromagnets. Phys. Rev. B 64, 024432 (2001).

12. A.M. Kosevich, B.A. Ivanov, A.S. Kovalev. Magnetic Solitons. Phys. Rep. 194, 117 (1990).

13. Y. Zhou, E. Iacocca, A.A. Awad, R.K. Dumas, F.C. Zhang, H.B. Braun, J. Akerman. Dynamically stabilized magnetic skyrmions. Nat. Comm. 6, 8193 (2015).

14. A.N. Bogdanov, D.A. Yablonskii. Thermodynamically stable "vortices" in magnetically ordered crystals. The mixed state of magnets. Sov.Phys. JETP 95, 178 (1989).

15. B.A. Ivanov, V.A. Stephanovich, A.A. Zhmudskii. Magnetic vortices: The microscopic analogs of magnetic bubbles. J. Magn. Magn. Mater. 88, 116 (1990).

16. S. Muhlbauer, B. Binz, F. Jonietz, C. Pfleiderer, A. Rosch, A. Neubauer, R. Georgii, P. Boni. Skyrmion Lattice in a Chiral Magnet. Science 323, 915 (2009).

17. X.Z. Yu, Y. Onose, N. Kanazawa, H.H. Park, J.H. Han, Y. Matsui, N. Nagaosa, Y. Tokura. Real-space observation of a two-dimensional skyrmion crystal. Nature 465, 901 (2010).

18. X.Z. Yu, N. Kanazawa, Y. Onose, K. Kimoto, W.Z. Zhang, S. Ishiwata, Y. Matsui, Y. Tokura. Near room-temperature formation of a skyrmion crystal in thin-films of the helimagnet FeGe. Nat. Mater. 10, 106 (2011).

ISSN 2071-0194. Ukr. J. Phys. 2019. Vol. 64, No. 10 
19. Ar. Abanov, V.L. Prokovsky. Skyrmion in a real magnetic film. Phys. Rev. B 58, R8889(R) (1998).

20. A.V. Bezvershenko, A.K. Kolezhuk, B.A. Ivanov. Stabilization of magnetic skyrmions by RKKY interactions. Phys. Rev. B 97, 054408 (2018).

21. M. Ezawa. Giant Skyrmions Stabilized by Dipole-Dipole Interactions in Thin Ferromagnetic Films. Phys. Rev. Lett. 105, 197202 (2010).

22. Y.Y. Dai, H. Wang, P. Tao, Y. Yang, W.J. Ren, Z.D. Zhang. Skyrmion ground state and gyration of skyrmions in magnetic nanodisks without the Dzyaloshinsky-Moriya interaction. Phys. Rev. B 88, 054403 (2013).

23. M. Schott, A. Bernand-Mantel, L. Ranno, S. Pizzini, J. Vogel, H. Béa, C. Baraduc, S. Auffret, G. Gaudin, and D. Givord. The Skyrmion Switch: Turning Magnetic Skyrmion Bubbles on and off with an Electric Field Nano Lett. 17, 3006 (2017).

24. A. Bernand-Mantel, L. Camosi, A. Wartelle, N. Rougemaille, M. Darques, L. Ranno. The skyrmion-bubble transition in a ferromagnetic thin film. SciPost Phys. 4, 027 (2018).

25. V.P. Kravchuk, D.D. Sheka, A. Kakay, O.M. Volkov, U.K. Röbler, J. van den Brink, D. Makarov, Y. Gaididei. Multiplet of Skyrmion States on a Curvilinear Defect: Reconfigurable Skyrmion Lattices. Phys. Rev. Lett. 120, 067201 (2018).

26. R.V. Verba, D. Navas, A. Hierro-Rodriguez, S.A. Bunyaev, B.A. Ivanov, K.Y. Guslienko, G.N. Kakazei. Overcoming the limits of vortex formation in magnetic nanodots by coupling to antidot matrix. Phys. Rev. Applied 10, 031002 (2018).

27. D. Navas, R.V. Verba, A. Hierro-Rodriguez, S.A. Bunyaev, X. Zhou, A.O. Adeyeye, B.A. Ivanov, K.Y. Guslienko, G.N. Kakazei. Route to form skyrmions in soft magnetic films. APL Mater. 7, 081114 (2019).

Received 22.01.09

К.Е. Заспель, Г.М. Вайсін, Б.А. Іванов

МАГНІТНІ ХІРАЛЬНІ СОЛІТОНИ

В ТОНКОПЛІВКОВОМУ НАНОКОНТАКТІ

З ЕЛЕКТРИЧНИМ СТРУМОМ, СТАБІЛІЗОВАНІ ПОЛЕМ ЕРСТЕДА

$\mathrm{P}$ е $з$ ю м е

Статичні магнітні солітони в тонкій плівці, такі як скірміон, являють собою метастабільні стани, які можуть бути стабілізовані за допомогою балансу обмінної взаємодії і різних релятивістських взаємодій. Одним з найбільш ефективних стабілізуючих членів є антисиметричний обмін поряд з іншими, такими як магнітостатичні взаємодії в обмежених структурах, а також у наноконтакті із струмом на тонкій феромагнітній плівці. В даній статті досліджено вплив струму наноконтакту на енергію як топологічних (Т-типу), так і нетопологічних (NT-типу) солітонів. Без антисиметричної обмінної взаємодії поле Ерстеда наноконтакту може стабілізувати обидва типи солітонів, NT-солітони є основним станом. При наявності антисиметричного обміну виникає критичний струм наноконтакту, при якому Т-солітон стає основним станом. 